\title{
Impact of Change in Business IT Alignment: Evaluation with CBITA Tool
}

\author{
Imgharene Kawtar ${ }^{1}$, Baina Salah ${ }^{3}$ \\ Mohammed V University \\ ENSIAS, Rabat \\ Morocco
}

\author{
Doumi Karim ${ }^{2}$ \\ Mohammed V University \\ FSJES, Rabat \\ Morocco
}

\begin{abstract}
Organizations introduce changes to adapt to an agile context in a turbulent environment. These change often have an impact on business and information technology. In most cases, the change impacts organizational elements that make the adaptation not well defined, leaving out elements that can lead to misalignment. The change is realized in this article as a project, which will impact all the Business-IT alignment elements. It is important to know the scope of the impact in order to make a complete adaptation. By reviewing the literature, we found that there is no work dealing with this aspect. To fill this gap, we determine the impact of the project on the organization by considering of Business-IT alignment and we proceed with a comparison of the AS IS model of the organization with the TO BE model which is the target to be implemented keeping the system aligned. Accordingly, we propose a metamodel for change by the impact of the project on the Business-IT alignment and a set of rules and algorithms to predict the impact and adaptation. To make these contributions operational, the implementation of Change Business-IT Alignment Tool and demonstrate its applicability through a case study of an urban agency.
\end{abstract}

\section{Keywords-Agility; business IT alignment; change; project}

\section{INTRODUCTION}

Today's businesses are facing rapid and dynamic changes in the business environment, making agility a critical step in achieving competitive advantage over competitors. At that time, when the importance of alignment for effective organizational performance was emerging is now well recognized and must be invoked: it is "Business IT Alignment" which is dedicated to providing an aligned system where the elements of a business are not communicated with each other. The world is spinning, not necessarily very round, but certainly faster and faster, and the man in the middle of it all, if he creates the conditions for this acceleration, must also face it. Change is the scale of any organization, when it's needed it affects all the elements of business IT alignment. The last one is defined and modeled on good theoretical [1], presented [2] and empirical [3] standards, but rarely who talks about agility or change with the alignment Business IT [4][6].

As cited during our previous research as there is little research into the dynamics of alignment, the old research before technology, digital transformation appears, R. Greenwood and C. R. Hinings [7] views alignment as a dying target on which organizations are heading, while S. L. Jarvenpaa and B. Ives [1] suggest that it should be examined as an emerging process. But today's environment is constantly changing, it continues to change, slowly or rapidly, but it is changing all the time. Even after an organization has achieved alignment, its environment would continue to change.

However, organizations may not be able to adapt their alignment models to changes in the external environment for one main reason: Too much emphasis on alignment may restrict the organization's perspective, impede the recognition of other perspectives, and reduce the ability to "recognize and respond to the need for change" [8]. Alignment facilitates short-term success, which leads to structural and cultural inertia, and inertia, in turn, leads to failure when market conditions suddenly change [9].

As a result, when organizations with a high level of alignment are faced with unexpected changes in industry conditions, they may find it difficult to respond with evolutionary changes. Revolutionary change would be more likely [10]. If the strategy or structure of the company is changed accordingly:

- Will the other elements be modified in a synchronized way to maintain alignment?

- Or would there be periods of poor alignment until the other elements are realigned?

During previous research, it was found that there is no study regarding the impact of change on business IT alignment, the comparative study of [11] concluded that few strategic alignment approaches address the impact of change, therefore this paper will demonstrate the impact of the project as a change to an aligned system.

The main objective of this article is to define and maintain over time, the model that will allow analysing the impact of the change and the change itself to maintain adaptability between elements and maintain Business IT alignment. In our previous research [12], agility was seen as a change with concrete and valid definitions. This paper will define change as a project, the impact of the project in an enterprise will involve the change in the elements of the Business IT alignment, and the goal is to define its changes by analyzing the study of the existing with the project to define the goal to be implemented without a misalignment. The analysis will be done through a project that will be modeled by rules involved with the Business IT alignment, a description of the study of the existing will be modeled by the Archimate language and a 
homogeneity will be done with the project to have a future model also modeled by the Archimate language which will be the future target to implement.

The objective is to integrate a new project in the company by analyzing the change that will impact the elements by comparing the study of the existing (AS IS) with the target to be maintained (TO BE) taking into consideration the modeling of the project and integrating the French state urbanization model as a repository of Business IT alignment. A 3-step process will be explained in the following sections. A process that will define that the project will be quantifiable for the company without damaging the Business IT alignment too much and by analyzing the change and the Business IT alignment elements impacting, then we have a prototype that will concretize this contribution and will be simulated by a case study of a Moroccan urban agency. Section 1 defines the work related between the Business IT alignment and an aligned project, Section 2 will define the approach that will be concretized by an AS IS model and a TO BE model using Archimate language, Section 3 elaborates the impact analysis of the change on an aligned system and finally a Section 4 that elaborates the CBITA prototype that will be validated by a case study of a Moroccan urban agency.

\section{RELATED WORK}

\section{A. Impact Change in Alignment Business IT}

The research [13] has mentioned that the relationship between agile changes largely affects all elements of business IT alignment; they have described that when there is a change in an entity that contributes to strategic alignment automatically it is all the entities that touch each other. Over the course of the literature, we find that the majority of the work concerning the changes that impact the system and not precisely the harmonization between the elements of the strategic alignment, these works are essentially in different domains including the modeling of company or the software architecture. For the latter, we examined the work of [14] Williams et al. provided a framework for assessing change characteristics (e.g., sources, types of change, granular effect, etc.) and their impact on architecture, [15] they also determine the different characteristics of agile change, they develop the sources of change and integrate them into the system. This paper concludes with a focus on the impact of agile change on business IT alignment, primarily: (i) change is triggered by sources as well as type of change, (ii) impact touches all the components and entities of a business IT alignment whether it is Bottom Up or Top Down.

\section{B. Alignment Business IT with an Aligned Project}

The selection of projects and initiatives is an area where Business IT alignment can be easily and quickly improved and where this work has a massive impact on organizational performance. Selection is often done by an executive committee and, because of this level of visibility, people tend to assume that the resulting project portfolio is aligned with strategy. It is not easy to lead a change in a company, especially when you know that a \% of projects are not supported, i.e. do not achieve their objectives. There are many indicators to be taken into account to start a transformation.
By focusing on the resources of the most strategically important projects, value is created with less risk. Therefore, projects that are aligned with strategy have a better chance of achieving their objectives. In other words, by measuring the Business IT alignment of projects and eliminating projects that are not aligned, these resources can be redirected to important projects that are in difficulty and turned into successful projects. Project prioritization is the basis for successful project implementation. This is where the alignment of projects to strategy requires.

Incorrect prioritization leads to the failure of projects and, ultimately, to the non-attainment of objectives. Properly conducted, a good project process will strategically align resource allocation decisions while achieving more successful projects. This seems to be reason enough to invest in improving the project prioritization process, but many organizations do not even realize that their current process is flawed; it has evolved over time, it worked last year and it will work next year.

The environment is constantly changing around us, and if we do not have a well-structured process to recognize and respond to change in a way that has broad management support, then the organization will fail to achieve its goals.

This is where we need to prioritize projects:

- Align projects with strategy;

- Compare the study of the existing (existing project) with the study to be implemented (future project) and;

- Analyse the impact of the project on the aligned system to quantify the projects that add value and the ones that do not.

To achieve this, an approach consisting of three important phases will be followed in the following section.

\section{Process for Impact a PRoject on AN Aligned SYSTEM}

The contribution will begin with an approach that consists of 3 important phases as shown in Fig. 1. It includes (1) a model of the study of the existing of the enterprise (AS IS) defined by the Archimate language that will be the first step, then (2) the project that is the source of change and after (3) the impact of this project on the study of the existing to have the model defined by the Archimate language that we want to put in place taking into consideration the Business IT alignment (TO BE) [16].

- Pre-requisites: Study of the existing 'AS-IS' using the of Urbanisation reference of the information system frame of the French government's;

- IT project modeling: Modeling of the project with different missions and processes of different levels of abstraction (same repository as AS-IS but with the project objectives);

- Change analysis: To determine if the project will be suitable for adoption by the AS-IS, a comparison is needed to determine the changes that will be supported. 


\section{A. Phase 1: AS -IS}

In this step, an "AS IS" is carried out on four levels, (1) the processes with high added value of the company, (2) the organization of the application architecture, (3) functional and flows, (4) the organization of the technical architecture.

The inventory identifies the points of difficulty and possible risks associated with the information system and/or the IT system. These risk points may be the subject of projects to be implemented as part of the improvement of the information system using the 'French State Urbanization reference system' [17].

Previous research on business IT alignment [18]-[20] ... has approached strategic alignment from a modelling and evaluation point of view with an appropriate result, but little research has been carried out on the evolution of this Business IT alignment with unforeseen events and changes.

This model [17] will be our referential on the existing as shown in Fig. 2, it is a French state urbanization model which in order to facilitate mutual understanding both of patrimony but also of initiatives and projects of transformation. It will allow us to list and classify the subjects concerned: data, applications, processes, but also software components or technical equipment, as the initiatives and transformation projects. The objective is to define and maintain for each viewpoint on the IS (strategy view, business view, functional view, application view, infrastructure view) a reference nomenclature that allows:
- Structure the assets and their transformation;

- Facilitate communication and understanding of heritage and projects;

- Definition of the existing technical architecture.

\section{B. Phase 2: Modelling Project}

The project will have its own modelling is presented in Fig. 3 independent of the AS IS model where we will define its strategy, its declination on the business processes and its declination on the functions of the information system and we will see further on if this project will have an added value by keeping the alignment between the strategy and the information system.

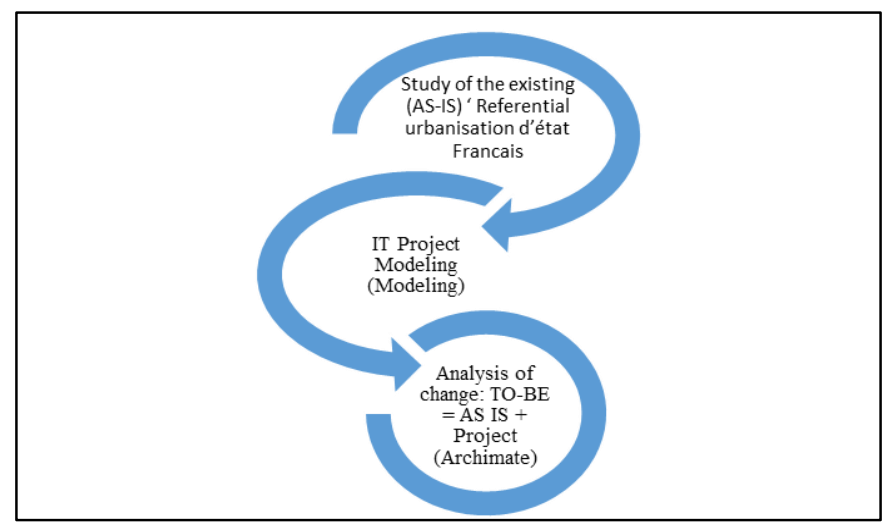

Fig. 1. Process of Impact of a Project on Business IT Alignment.

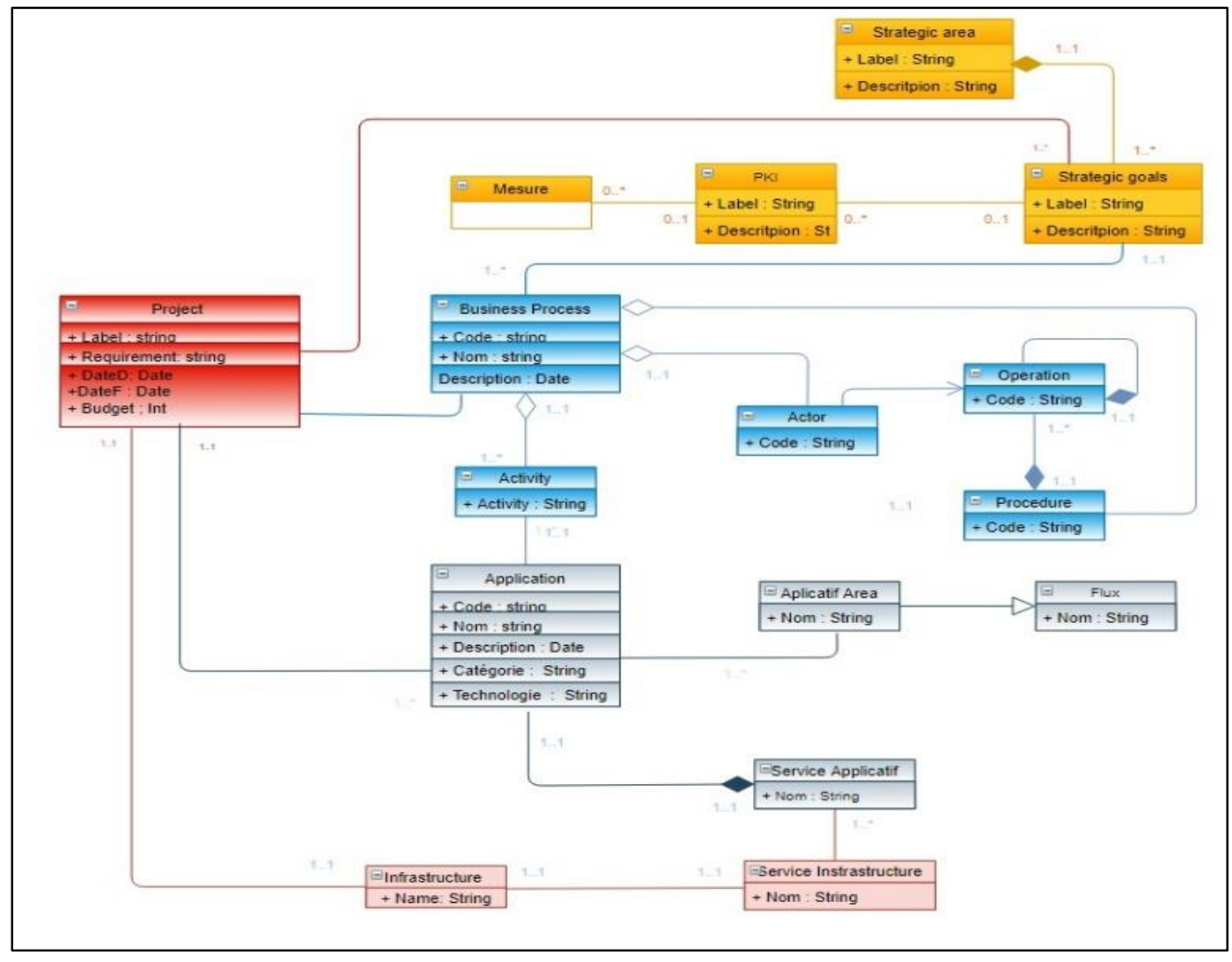

Fig. 2. French State Urbanization Reference Information System. 


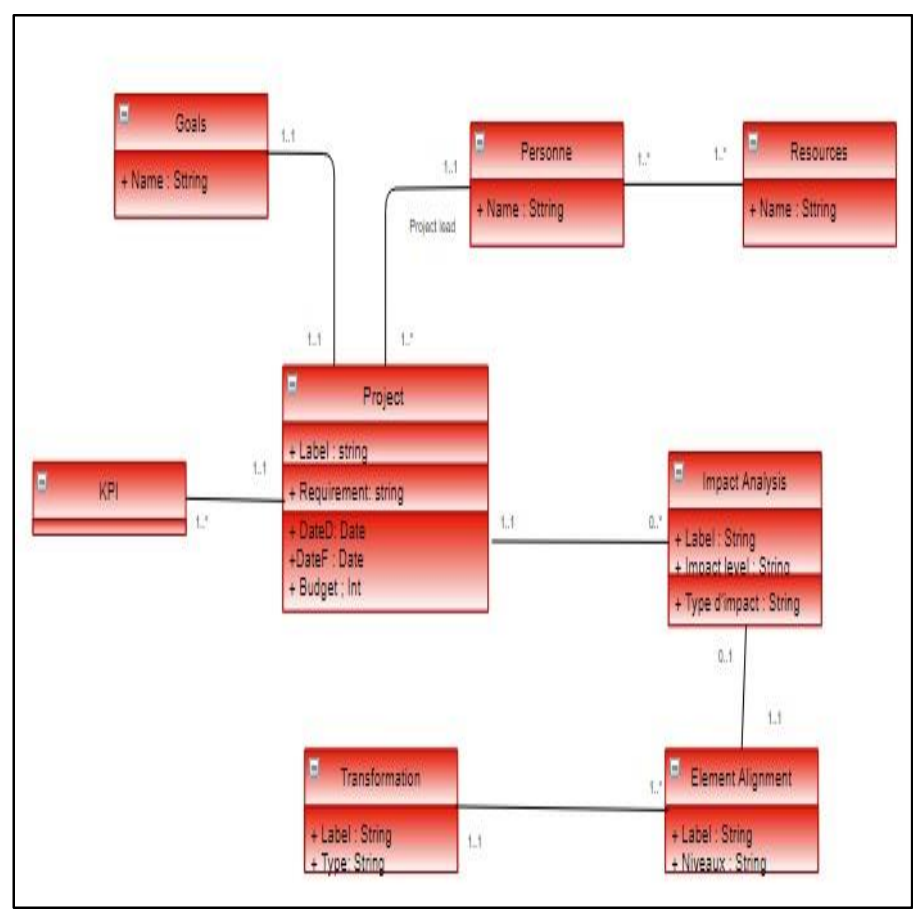

Fig. 3. Project Modeling.

The model will be characterized by a modeling process is presented in Fig. 4 aimed at obtaining an acceptable solution from the computer system. The solution finally selected is not obtained in a single iteration. Several steps are necessary; these successive steps allow the level of detail of the system to be refined. The first steps give a very large vision and allow to progress in the understanding of the problem.

\section{Phase 3: To be - Change Analysis}

Before starting a project, an impact analysis should be conducted in order to identify all the consequences of a change on employees, processes, business functions, other ongoing projects, etc. as mentioned in Fig. 5.
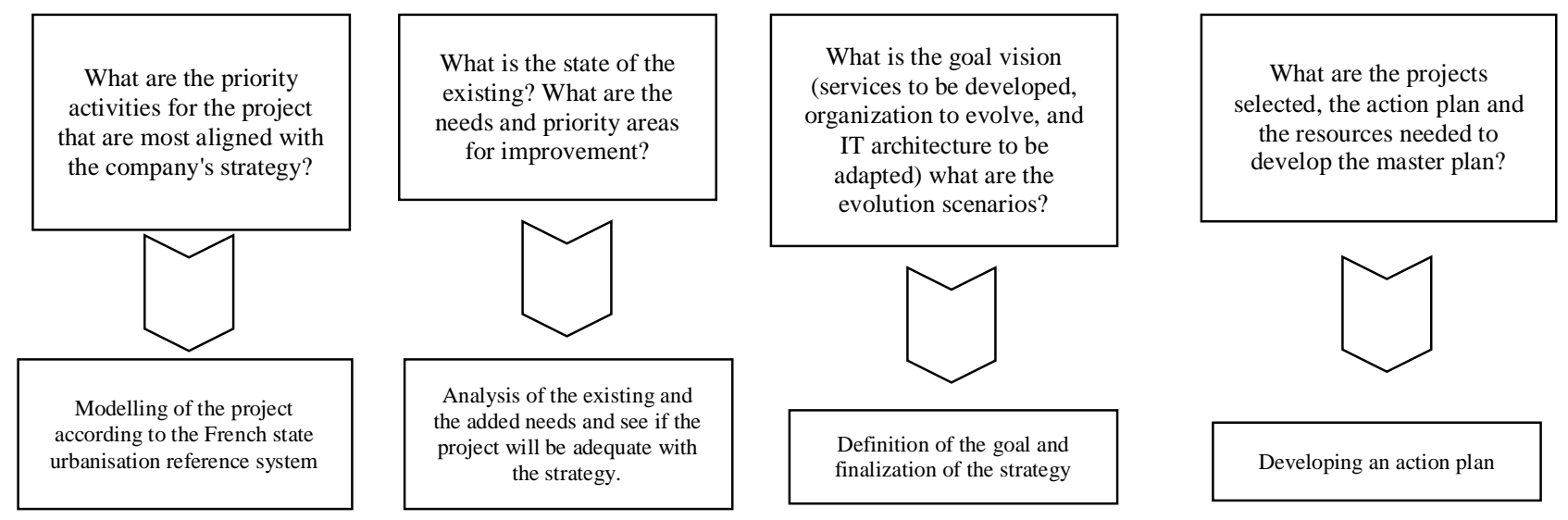

Fig. 5. Change Analysis.
Fig. 5 shows the following steps:

- Expression of needs: The need expression phase involves identifying the request, clarifying and specifying the need;

- Feasibility study: The feasibility study concerns the technical, financial and availability of means for the project;

- Action plan: Defining, organizing, planning and implementing all the means to achieve the set objectives.

A mechanism must therefore make it possible to define "what should be done" (i.e. in compliance with the rules of business IT alignment) and compare it as soon as possible with "what can be done" (i.e. in compliance with cost and time constraints).

Phase 3 will be formalized by the ArchiMate language [13] which is relatively a new standard for high-level enterprise architecture modeling. It provides a comprehensive modeling language that has been formally developed to describe and analyse the architecture of an enterprise. It provides a meta-model and graphical notations to visually describe and analyse the three layers of EA (Business, Application and Infrastructure), their relationships and dependencies. The main advantage of ArchiMate is its ability to cover all layers of the enterprise, a single language for all layers and viewpoints of the business, from the business to the technical foundation.

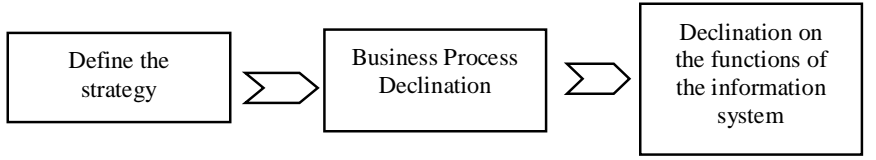

Fig. 4. Strategy of a Project Modeling.
IT architecture to be adapted) what are the

Definition of the goal and finalization of the strategy 


\section{The Impact of the Aligned Project on an Aligned SYSTEM}

Alignment takes place at the business and functional levels, from a technological and organizational point of view presented in Fig. 6. Through projects, the information system tries to structure itself in an organized way [21]. From an economic point of view, the application of an investment typology at the project portfolio level makes it possible to respond to two complementary management challenges:

- A gradual realignment of the value provided: a strategic opportunity approach may emerge for the business and IT.

- Taking into consideration the nature of the projects and their specificities in order to compare them in a common framework: response to the prioritization difficulties frequently encountered (between strategic projects and infrastructure projects, for example).

The impact of a project includes all related information in terms of resources, schedules, budgets, benefits and risks. It can also include other information related to the nature of the company's activity or principles related to the Business IT alignment of all levels of abstraction. The project manager will define the cost and quality of the project and the duration. The business IT Alignment Architect will define the limit and weight of the Business IT alignment entity elements and will make a comparison between AS-IS and TO-BE model from Archi tool to distinguish between projects that are supported and projects that are not supported by the project management system presented in Fig. 7.

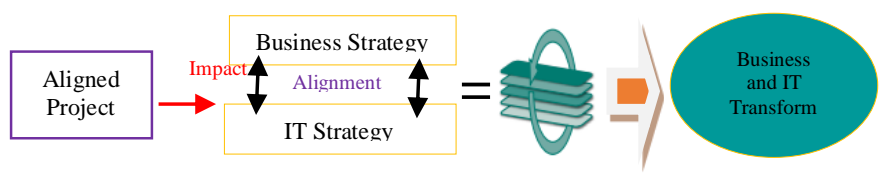

Fig. 6. Impact of an Aligned Project.

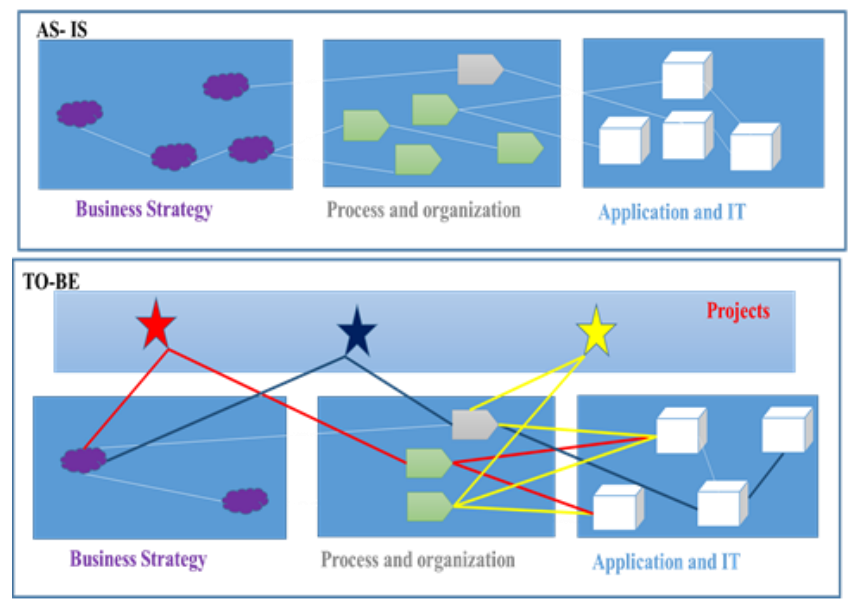

Fig. 7. Impact of an Aligned Project on an Aligned System.

\section{Case Study: Urban Agency Implementing A GEOGRAPHIC INFORMATION SYSTEM}

The process explained in the previous sections will be validated by the case study of an Urban Agency implementing a Geographic Information System.

\section{A. Study of the Existing}

Several interviews were held with the various stakeholders of the basic departments of the Laayoune-Sakia el Hamra Urban Agency with a view, on the one hand, to detail the areas to be computerized and, on the other hand, to collect the expected needs of the GIS to be set up.

These interviews focused on the following points:

- Existing organization and procedures,

- Data produced by the Agency: nature and media of the data;

- Existing alphanumeric and cartographic data;

- Existing in terms of available databases and applications;

- Existing in terms of IT platform;

- Needs in terms of data and processing;

- Existing in terms of completed project.

The analysis of the existing is part of the detailed analysis phase of the project. This step, which is essential to any project start-up, helps to define and encourage the creativity of the team in charge of developing the new GIS.

Fig. 9 illustrates the study of the existing which is modelled by the Archi tool, which is a visual design and modelling tool for creating ArchiMate models and modelling designs. The AS IS will be formalized to understand the concept of the study of the existing.

The export of the AS IS model in CSV is presented in Fig. 8 by the Archi tool will help us to make a comparison with the CSV of the TO BE model to feed the change impacted by the project, a prototype will be created to compare the $2 \mathrm{CSV}$ files and determine the change that was achieved.

\begin{tabular}{|c|c|c|}
\hline Type & Name & Documentation \\
\hline bd7f4b0d-f1d1-4f5a-b578-atArchimateModel & ASISF & \\
\hline bb72f94a-2db4-4024-8bdo-3 BusinessActor & Agent & \\
\hline 5437a090-b03a-44a4-9426-fc BusinessActor & DAJF & \\
\hline da1b3d65-c0e2-4478-99b4-b BusinessFunction & AccessibilitÃ@s aux donnÃ@es & \\
\hline b00a9c3f-6c53-404c-a276-bb BusinessFunction & Gestion des dossiers & \\
\hline f30198ae-0393-4c7c-86ee-19 BusinessFunction & Gestion des Infractions & \\
\hline e56ec39f-d7a1-49e1-9586-bı BusinessProcess & Accesibilitã (@s aux donnÃ@es & \\
\hline ae385912-561e-4eb3-a5a1-fi BusinessProcess & Commission & \\
\hline Bae0b003-e33f-4066-ae88-7 BusinessProcess & Convocation & \\
\hline e4adf49f-054a-4826-80cd-49 BusinessProcess & Dispatching & \\
\hline fa16a719-cfca-422d-8367-e2 BusinessProcess & Documenter les donnÃCes & \\
\hline 8c48d354-f1b2-4ddf-8ee2-2iBusinessProcess & Elaborer les demandes & \\
\hline 22952cd4-3749-4f64-a5e0-bc BusinessProcess & Enregistrement & \\
\hline fc4ea7c5-bf2c-466f-9a1f-3ac BusinessProcess & \multicolumn{2}{|c|}{ Envoi de la lettre et copie des PV pour la signature par le DG } \\
\hline
\end{tabular}

Fig. 8. AS IS Model Export in CSV. 


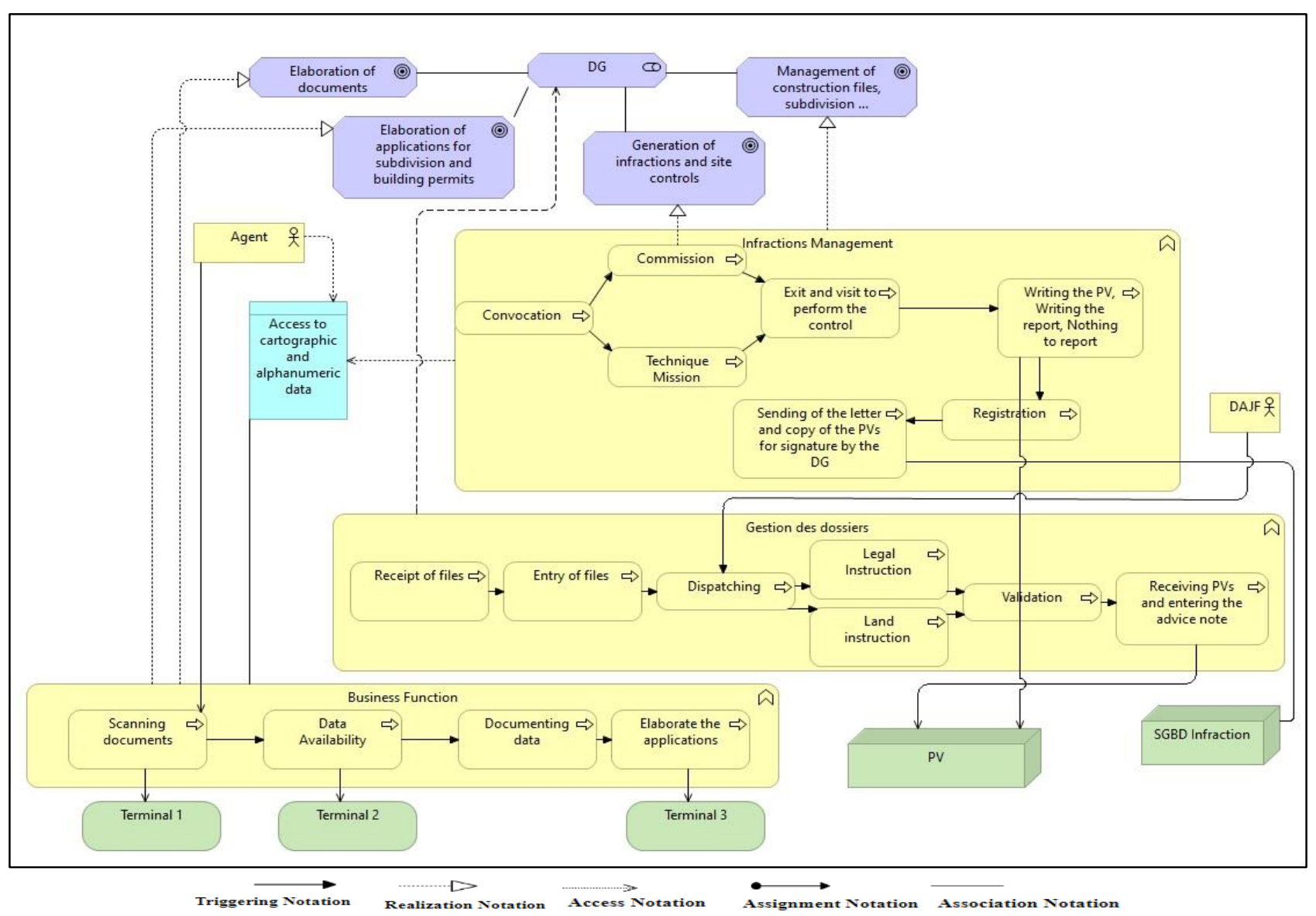

Fig. 9. Modeling of the GIS Project with AS IS Model.

\section{B. Modeling of the GIS Project}

The Urban Agency, as a public agency in charge of Urban Planning and technical assistance to its partners in its territorial area, is required to manage on a regular basis a large amount of information from different sources, natures and media.

The implementation of a WEB GIS (Geographical Information System) platform and online services, on behalf of the Urban Agency, with the aim of controlling the information possessed and acquired, rationalizing the archiving and updating of information and accessing various statistics and carrying out spatial analyses.

The GIS tool presented in Fig. 10 must ensure the following functionalities: information, production and decision, it must cover all the basic procedures relating to the operation of the Urban Agency of Laayoune. This system must allow the automation of its procedures, support processes and business processes. The technical specifications relating to the implementation of this system must meet the overall constraints indicated below:

- Consultation and quick reading of urban planning documents and regulatory databases;

- The delivery of the information note;
- The automatic generation of monitoring and historical records of activities at the level of plots and specific study areas;

- The facility of exchanges with the various partners and customers by the GIS-WEB; (results of the files, information notes...);

- The electronic management of documents;

- Management of claims.

The proposed solution presented in Fig. 10 must respond precisely to the specific needs of the Administration in terms of data exploitation and functional organization. In particular, it must be based on an internationally recognized GIS \& DBMS solution that can guarantee interoperability, data sharing and ensure the durability and evolution of the solution. The final objective being: the achievement of the following missions:

- Electronic document management;

- E-note;

- E-reclamation;

- E- infraction;

- Geoportal for urban planning. 


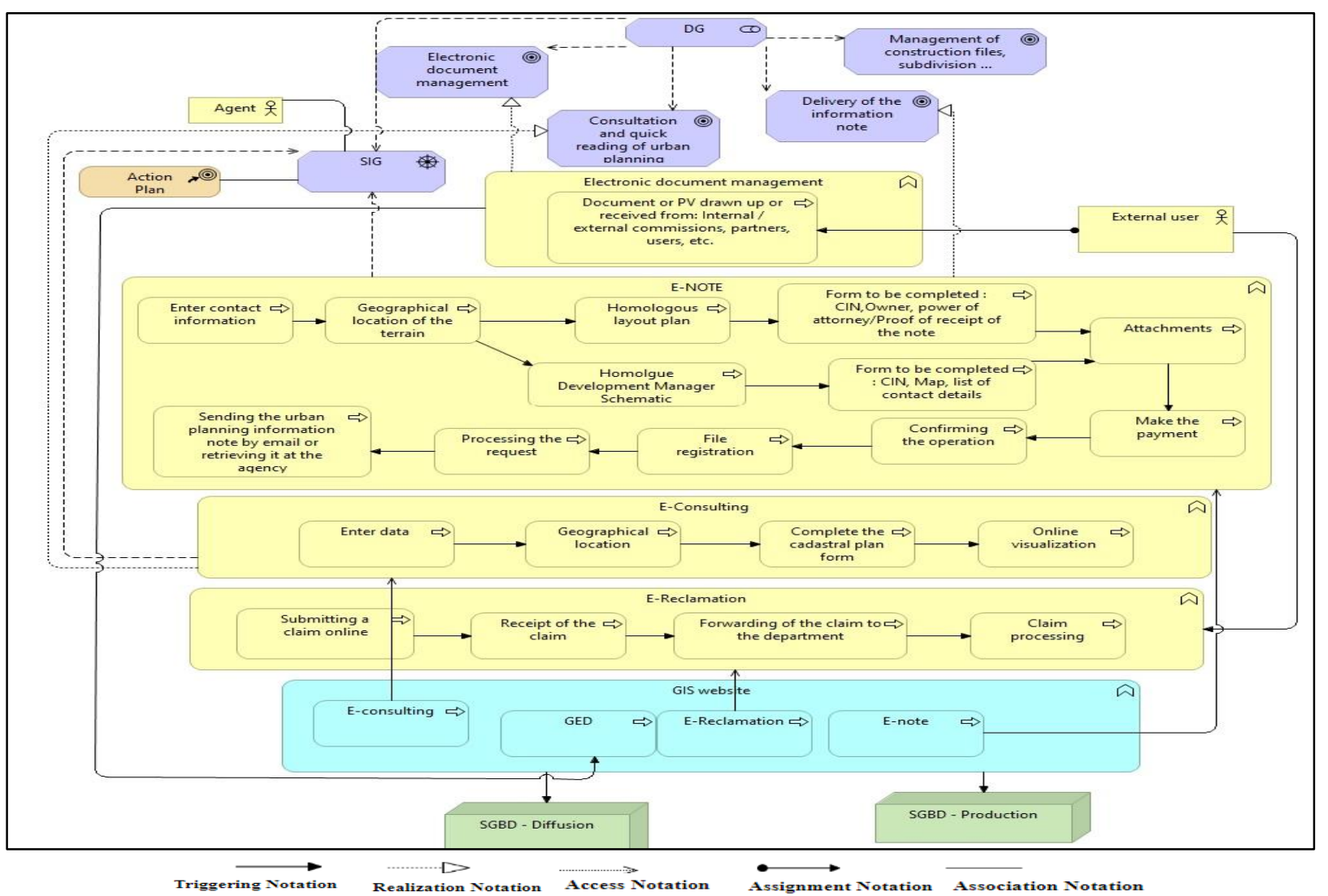

Fig. 10. Modeling of the GIS Project.

\section{Analysis of Change: TO BE}

After the analysis on the basis of the 'Special Conditions' which was transmitted by the agency, the impact of the project on the study of the existing will transform all the objectives of the agency to different modules developed in the GIS:

The TO BE model is presented in Fig. 12 will be determined as follows:

- The proposed GIS platform must be based on a secure Intra/Internet solution for its deployment and operation;

- The solution must make use of the digital mapping tools available, which the contractor undertakes to acquire on behalf of the management, and must take into consideration all the applications and databases already deployed within the management and at the supervisory Ministry (possibility of integrating the ministerial frameworks to save time and guarantee the reliability of the data provided).

- The solution must ensure the integration, management, centralization, in databases and the updating, if necessary, of all the data involved in the business, such as cartographic, photogrammetric, remote sensing and socio-economic data;

The system will have to take into account all the information essential for its implementation by integrating georeferenced or non-geo-referenced information (documentation, authorization file, Image...) with their metadata to define all the technical and descriptive information (digitization scale, accuracy...);

Objectives are added to complete the overall goal of the agency, these objectives are aligned with business processes that will be modulated by activities, using the WEB GIS platform to disseminate all the services that will be handled by the agency's agents. After the modelling of the impact of the project on the study of the existing, the 'TO BE' model will be imported in CSV presented in Fig. 11 format in order to execute the comparison with the CSV file of the study of the existing 'AS IS' mentioned above to determine the acquired transformations and to analyse the change. The following section will demonstrate the prototype that supports this comparison.

\begin{tabular}{|c|c|c|}
\hline Type & Name & Documentatio \\
\hline 0d7f4b0d-f1d1-4f5a-b57 ArchimateModel & TO BEF & \\
\hline 9b72f94a-2db4-4024-8bc BusinessActor & Agent & \\
\hline 5437a090-b03a-44a4-942 BusinessActor & DAJF & \\
\hline dafd5d87-84e3-4d39-ab، BusinessActor & Responsable du dÃ@ partement de Gestion ur & rbaine \\
\hline 8635c0d2-57e3-468e-94f BusinessActor & Utilisateur externe & \\
\hline 347bca8b-cfe0-44a1-8b3 Businessfunction & E-Consultation & \\
\hline a7f1653f-2081-47c8-8bbi Businessfunction & E-NOTE & \\
\hline 1549a78a-83e9-44a1-bf7 Businessfunction & E-RÃ@clamation & \\
\hline f30198ae-0393-4c7c-86e BusinessFunction & Gestion des Infractions et Controles & \\
\hline 358d741c-9699-4c3e-845 Businessfunction & Gestion Ã@IÃ@ctronique des documents & \\
\hline 000a9c3f-6c53-404c-a27t Businessfunction & IntÃ@gration Gestion de dossier dans LE SIG & \\
\hline 0c656d51-9606-4fa2-a93 Businessfunction & Sauvegarde Des documents & \\
\hline afaa5fa9-5b93-4d7e-bc1 BusinessProcess & Archivage dans une base de donnÃ $\odot$ es & \\
\hline 08a03972-c884-4fa2-bfcs BusinessProcess & Confirmation de l'opÃ@)ration & \\
\hline 2340be5e-5852-4ecc-90; BusinessProcess & Diffusion de la rã@clamation au dÃ@ parteme & \\
\hline
\end{tabular}

Fig. 11. To be Model Export in CSV. 


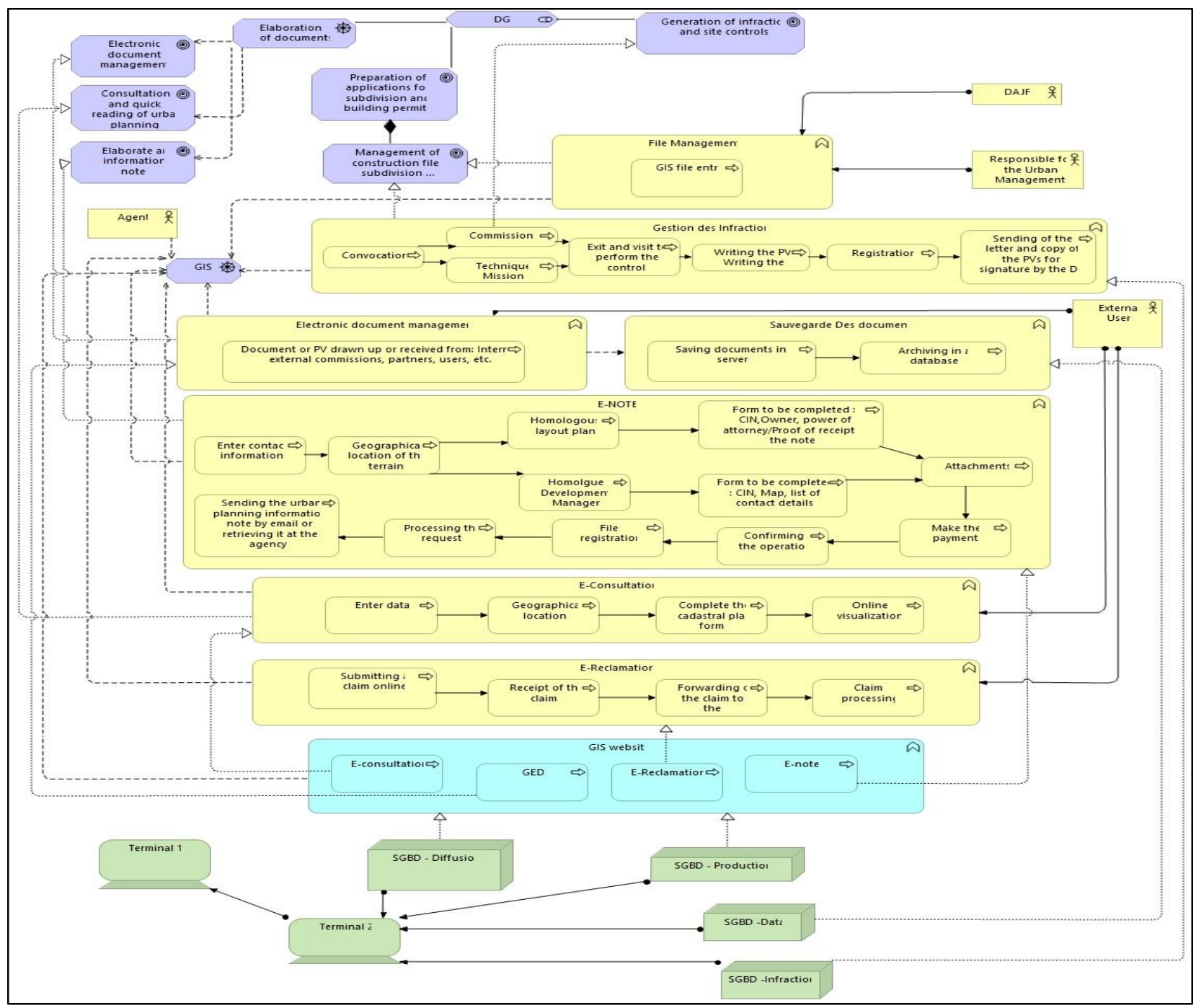

Fig. 12. Modeling of the GIS Project with AS IS Model = TO BE.

\section{Change in Business IT Alignment (CBITA) ToOL}

The CBITA (Change in Business IT Alignment) Tool developed to support change evaluation by comparing CSV files exported from the AS IS and TO BE modeling by the Archi tool proposed in the previous section.

The goal of this prototype is to provide a comparator by general algorithms that gives us the result of the evaluation of the impact of Project (Strategic Project Objectives and IT Project Objectives) on the study of the existing (an aligned system $=$ strategic objectives aligned with the IT objectives) by presenting the changes by entity of alignment, the modifications made, the redundancy, the deletion or addition of entities and to clarify the good project that will be eligible for the study of the existing. This Tool will allow the users (it can be the project manager, the strategic alignment architect ...) to know the appropriate alignment model for the study of the existing in relation to the project and to define the set of analysis of changes that the project can establish to the whole existing aligned system. This tool therefore allows: Fig. 13 show the comparison between 2 CSV files (AS IS and TO BE) to analysis the change, the Fig. 14 the evaluation of the alignment through algorithms to determine the number of changes between the different entities of Business IT alignment as also the exact change between the entities, and finally the Fig. 15 defines a graph which qualifies the best project by change (modification, deletion, addition). 


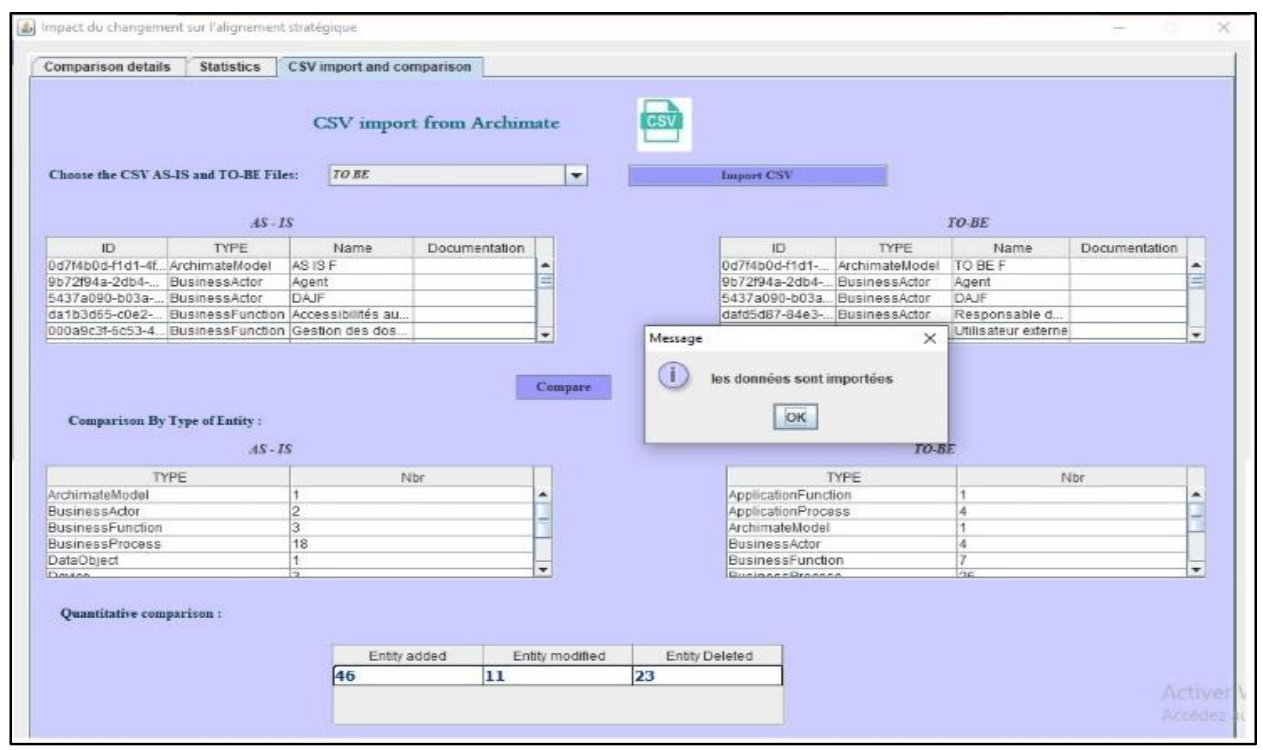

Fig. 13. Comparison between CSV Files = AS IS and TO BE.

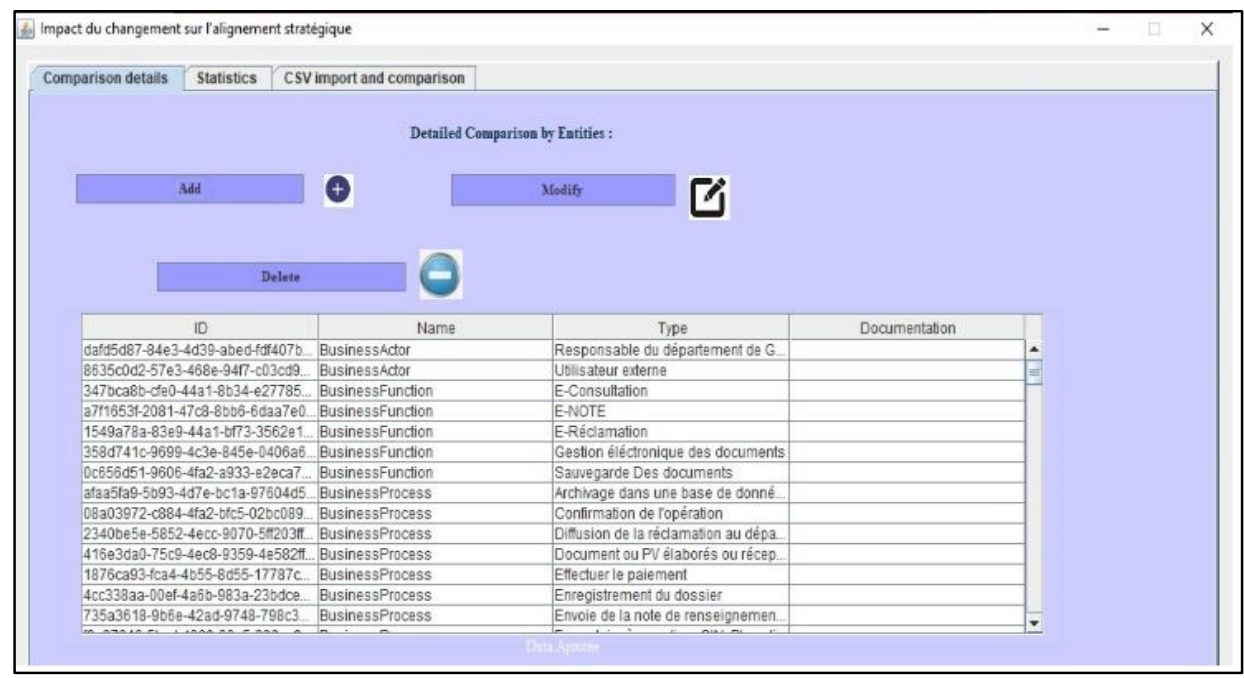

Fig. 14. Analyses of Change between the CSV Files.

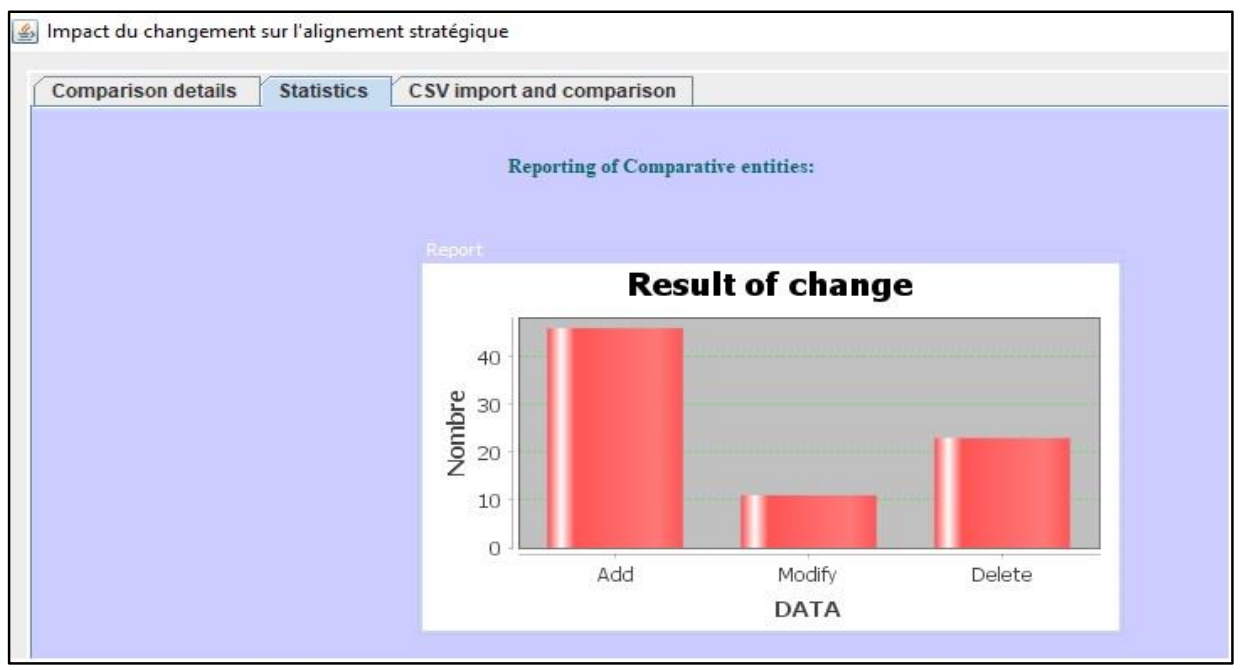

Fig. 15. Graph Illustrating the Impact of Change. 


\section{CONCLUSION AND FUTURE DIRECTION}

To conclude, this was possible to determine the impact of a project on an aligned system. A process was to be carried out to analyse the change in the impact of a project on an aligned system. The conclusion is that when a change affects the aligned system, it is all the system which changes: objectives change, business processes, activities, information system, technical infrastructure all change, so when a change affects the system automatically the whole system changes. The main objective of the contribution despite the change we must keep the strategic alignment. Future work will focus firstly on comparing the approach detailed previously with the other approaches in order to benefit from the comparative criteria. Secondly, we will try to add to the CBITA approach the option of choosing between several projects to demonstrate the most quantifiable project for an aligned system.

\section{REFERENCES}

[1] S. L. Jarvenpaa and B. Ives, "Organizing for Global Competition," Decis. Sci., vol. 24, no 3, p. 547-580, mai 1993.

[2] Andrew H. Van de Ven and Robert Drazin, "The Concept of Fit in Contingency Theory," p. 71, 1985.

[3] D. Miller, "Environmental Fit versus Internal Fit," Organ. Sci., vol. 3, no 2, p. 159-178, mai 1992.

[4] Tallon and A. Pinsonneault, "Competing perspectives on the link between strategic information technology alignment and organizational agility: insights from a mediation model," Mis Q., vol. 35, no 2, p. 463486, 2011.

[5] O. Avila and K. Garcés, "Change management support to preserve Business-Information technology alignment," J. Comput. Inf. Syst., vol. 57, no 3, p. 218-228, 2017.

[6] O. Avila and K. Garcés, "Change management contributions for business-IT alignment,", in International Conference on Business Information Systems, 2014, p. 156-167.

[7] R. L. Thompson and C. L. Iacovou, "Information technology, critical success factors and organizational performance of small firms: a causal modelling approach,", School of Business Administration, University of Vermont, Burlington, VT, Working Paper WP-MIS-93-006-1., 1993.

[8] D. Miller, “Configurations Revisited," Strateg. Manag. J., vol. 17, no 7, p. 505-512, 1996.

[9] M. L. Tushman and C. A. O'Reilly, "Ambidextrous Organizations: Managing Evolutionary and Revolutionary Change," Calif. Manage. Rev., vol. 38, no 4, p. 8-29, juill. 1996.

[10] R. Greenwood and C. R. Hinings, "Understanding Radical Organizational Change: Bringing Together the Old and the New Institutionalism," Acad. Manage. Rev., vol. 21, no 4, p. 1022-1054, oct. 1996.

[11] K. Imgharene, K.doumi, and S.Baina, "Impact of Agility on the Business IT Alignment Conference the International Symposium on Business Modeling and Software Design," BMSD, 2017.

[12] K. Imgharene, K. Doumi, and S. Baina, "Proposal model of change for Business IT Alignment," Procedia Comput. Sci., 2019.

[13] K. Imgharene, K. Doumi, and S. Baina, "Toward a model for agility and Business IT Alignment," BDCA, avr. 2018.

[14] B. J. Williams and J. C. Carver, MSU-081216 "Characterizing Software Architecture Changes: A Systematic Review,"

[15] R. Sartori, A. Costantini, A. Ceschi, and F. Tommasi, "How Do You Manage Change in Organizations? Training, Development, Innovation, and Their Relationships," Front. Psychol., vol. 9, mars 2018.

[16] The Open Group “ArchiMate 3.1 Specification,” p. 206, 2019.

[17] Etat Francais "Cadre commun d'urbanisation du SI de l'Etat Francais," 2016.

[18] K. Doumi, S. Baïna, and K. Baïna, "Modeling Approach Using Goal Modeling and Enterprise Architecture for Business IT Alignment," in Model and Data Engineering, sept. 2011, p. 249-261.

[19] A. Etien, "Ingénierie de l'alignement: concepts, modèles et processus: la méthode ACEM pour l'alignement d'un système d'information aux processus d'entreprise," Paris 1, 2006.

[20] L.-H. Thevenet, "Proposition d'une modélisation conceptuelle d'alignement stratégique: La méthode INSTAL," Université PanthéonSorbonne-Paris I, 2009.

[21] A. Asil, "Design and Implementation of Strategic Agility Evaluation Model with Structural Equation Modelling Approach," Acad. Strateg. Manag. J., févr. 2019, Consulté le: juill. 26, 2019. 